Case Report

\title{
Synchronous Lung Cancers: When Same Histological Types Feature Dif- ferent Molecular Profiles and Response Phenotypes
}

\author{
Giulia M Stella ${ }^{\varpi}$, Francesca Cemmi, Simona Inghilleri, Michele Zorzetto, Maurizio Luisetti and Ernesto \\ Pozzi
}

Department of Hematological, Pneumological and Cardiovascular Sciences - Section of Pneumology, University and Fondazione IRCCS Policlinico San Matteo 27100 Pavia, Italy

Corresponding author: Giulia M Stella, MD PhD, Department of Hematological, Pneumological and Cardiovascular Sciences - Section of Pneumology; University and Fondazione IRCCS Policlinico San Matteo 27100 Pavia -Italy; phone: +39 0382 503369, FAX: +39 0382 502719, e-mail: g.stella@smatteo.pv.it

(C) Ivyspring International Publisher. This is an open-access article distributed under the terms of the Creative Commons License (http://creativecommons.org/ licenses/by-nc-nd/3.0/). Reproduction is permitted for personal, noncommercial use, provided that the article is in whole, unmodified, and properly cited.

Received: 2011.07.13; Accepted: 2011.09.21; Published: 2011.09.23

\begin{abstract}
We discuss the case of synchronous bilateral lung cancers which feature the same histological phenotype and a different EGFR mutational profile. Both histological and molecular characterizations were performed on specimens derived thorough CT-guided fine needle aspiration. A first-line chemotherapy was unsuccessful. Subsequent objective response to the EGFR inhibitor Erlotinib was clearly coherent with the sequencing data and the mutated nodule was effectively reduced (> 50\%) after therapy, while the lesion assessed as EGFR wild type featured a slight response. This report has two relevant implications. It points out that in case of multiple malignant lesions at time of diagnosis, molecular profiling should be as extensive as possible and it might contribute to clarify the association between the lesions found. Besides the molecular analysis on cytology specimens could identify an accurate and safe diagnostic approach for clinical use.
\end{abstract}

Key words: synchronous bilateral lung cancer

\section{Introduction}

Correlation between mutations in cancer alleles and drug response is a key point to identify drugs or drug combinations that match the genetic profile of individual tumors. The identification of genetic determinants of drug response, by routine diagnostic approaches, is thus a clear priority of translational oncology. In Non-Small Cell Lung Cancer (NSCLC) genetic lesions affecting the Epidermal Growth Factor Receptor (EGFR) pathway act as predictive markers of response to small inhibitors ${ }^{1}$. Inappropriate EGFR overactivation is mainly consequent to somatic mutations occurring in those sequences which encode for the receptor tyrosine kinase (TK) domain ${ }^{2}$. EGFR amplification (detected by FISH in $20-40 \%$ of NSCLCs, according to different studies) seems to add a gain in response rates to Gefitinb and Erlotinib 3,4,5. On the other hand, mutations affecting the EGFR downstream transducers and mainly the KRAS oncogene have emerged as highly specific negative predictors of response to single anti-EGFR agents ${ }^{6}$.

\section{Case}

Here we describe the case of a 71 years old, currently smoker, Caucasian man who came under our observation due to the occasional detection by standard chest $X$ ray of a right pulmonary mass. During hospitalization, the patient underwent a total body CT scan that showed the presence of two solid parenchymal lesions: the first affecting the upper right lobe and a second nodule at the lower left lobe; 
no mediastinal and extrathoracic masses were detected. The subsequently performed endoscopical examination did not allow conclusive findings. The patient was then addressed to fluoroscopic CT-guided fine needle aspiration (FNA) of the two lesions. In both cases the cytological analysis (Fig. 1) was consistent with adenocarcinoma, displaying a TTF-1 and p63 positive immunohistochemical profile. A diagnosis of bilateral synchronous NSCLC (adenocarcinoma) was thus formulated. In order to evaluate the EGFR/KRAS mutational profile, tumor genomic DNA from formalin-fixed paraffin-embedded (FF-PE) corresponding samples was extracted and sequenced. Interestingly two different EGFR profiles were unveiled. Indeed we found that the right lesion carried the EGFR L858R somatic change, while no EGFR mutations were detected by sequencing genomic

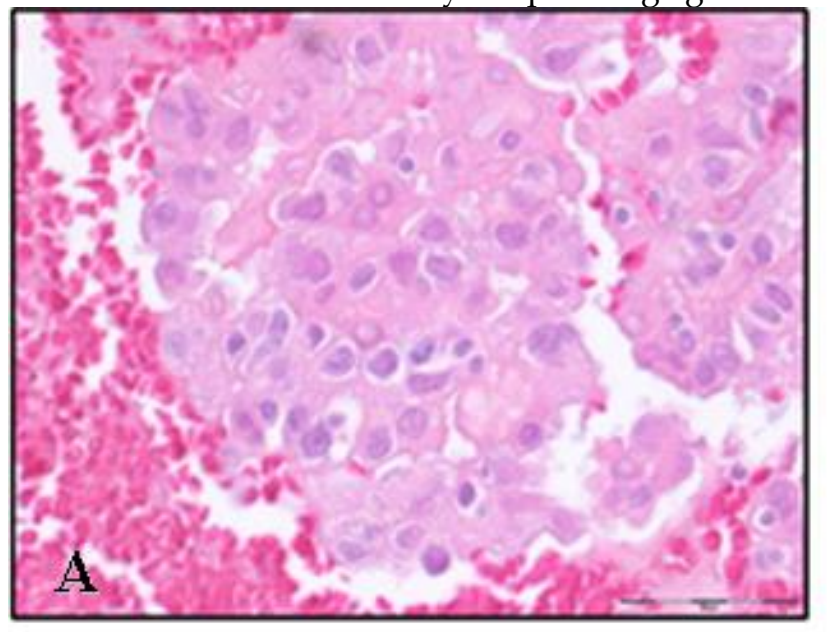

DNA extracted from the left nodule. Absence of EGFR amplification was documented by FISH analysis on both lesions. Besides the two masses harboured wild type KRAS sequences.

On this evidence, the patient underwent a first line platinum-based chemo (platinum-pemetrexed) but a slight disease progression was documented subsequent to 4 cycles of treatment. For that reason the patient was then treated with Erlotinib 150 $\mathrm{mg} /$ die. After 6 months of treatment the EGFR mutated lesion displayed a volume reduction of more than $50 \%$; the controlateral nodule showed a less but still significant $(15 \%)$ reduction in both diameter and density (Fig.2). Overall CT scans were performed as a control after therapies and objective response was evaluated according to RECIST criteria ${ }^{7}$.

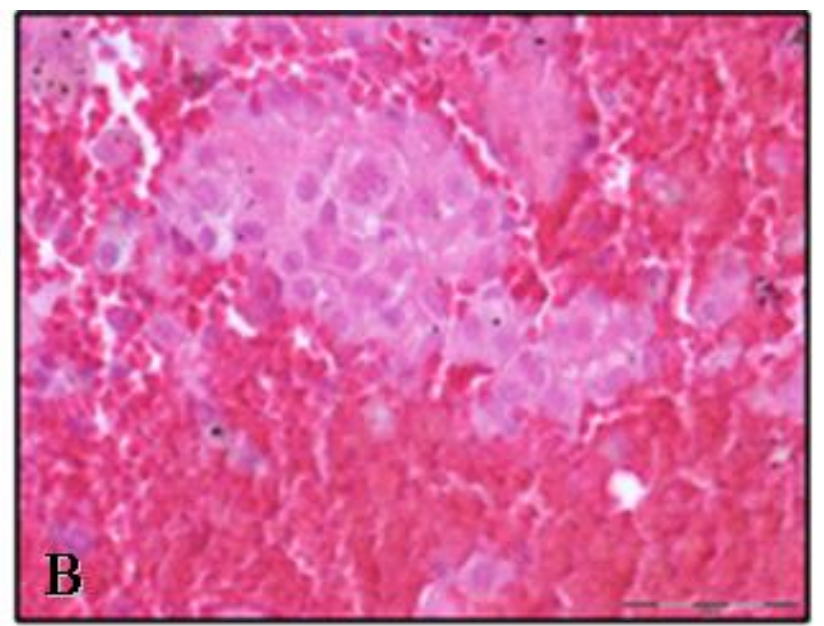

Figure I Formalin-fixed paraffin-embedded (FF-PE) samples of CT-guided fine needle aspiration of both the right (A) and the left (B) nodule (Hematoxylin and eosin stain, 40X.)

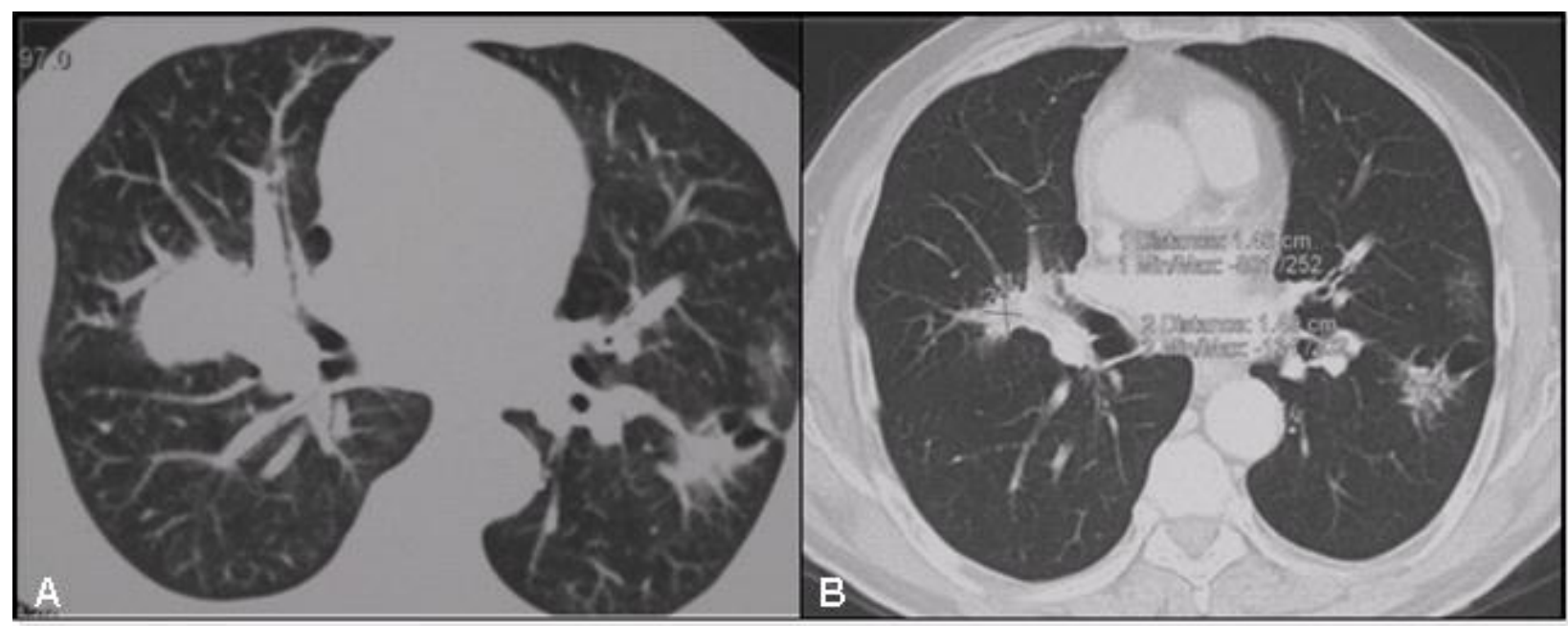

Figure 2. Evolution of tumor lesions after 6 months therapy with erlotinib as documented by $C T$ scan. Panel A: thoracic CT scan, after chemotherapy. Panel B: thoracic CT scan after 6 months of therapy with erlotinib, $150 \mathrm{mg} / \mathrm{die}$. 


\section{Discussion}

The above discussed case seems worth to be reported since it allows relevant some clinical considerations.

Interestingly, the lesion that was assessed as EGFR wild type - by sequencing FNA cytology specimen- actually displayed a slight response to the EGFR inhibitor. This behavior could be coherent with the fact that some responses to EGFR TKIs have been described also in EGFR wild type tumors ${ }^{8}$. In addition, it could be hypothesized the presence of an EGFR-mutated subclone. From this perspective, it should be note that direct sequencing might not be sensitive enough to detect low frequency of mutated EGFR and KRAS even though it remains at the present among the most accurate approaches to mutational analysis for clinical running.

A second key point is represented by the fact that routine histochemistry classified these tumors as two independent synchronous lung cancers. However based on their mutational profile, a potential chronology and a metastatic progression could be hypothesized. Indeed preclinical data suggest that EGFR mutations occur as an early event during NSCLC onset ${ }^{9}$. Besides it is known that the molecular status of EGFR/KRAS may change during the distant spreading of NSCLC 12,10 and a discordance between EGFR mutations in primary tumors and their corresponding lymphatic and distant metastases has been already reported $11,12,13,14$. The heterogeneity found in this case has relevant clinical implications since it is reflected in disease staging and therapy: if the putative second lesion was a metastasis, that meant a stage IV - not resectable - disease, whereas if it was a synchronous primary tumor, it was potentially resectable $^{15}$. Although an exhaustive immunoistochemical analysis could be helpful to clarify the association between the two lesions, this case undoubtedly demonstrates that regardless histochemistry, therapy should be tailored to the molecular profile and that a molecular profile can be linked to a clinical phenotype.

A third issue is related to the opportunity to routinely perform EGFR/KRAS mutational profile in front of ADKs aroused in males and active smokers. From this perspective, this report provides clear evidence for a need to a complete EGFR/KRAS molecular profiling for all lung ADKs.

Finally this clinical report underlines the role of molecular diagnosis on cytological tumor sample as a routinely safe and accurate approach. Coherently recent reports confirm the feasibility of these techniques in samples derived from US-guided fine nee- dle biopsies 16,17 . However it should be noted that although mutational analysis on small sized samples may underestimate molecular heterogeneity that usually characterizes lung cancer and that, consequently, the fraction of the mutated EGFR might be kept in consideration to define to what extent a lesion is mutated and consequently objectively sensitive to anti EGFR therapy.

\section{Conflict of Interest}

The authors have declared that no conflict of interest exists.

\section{References}

1. Herbst, RS, Heymach JV, Lippman SM. Lung cancer. N Eng J Med 2008; 359 (13):1367-80

2. Sharma SV, Bell DW, Settleman J, et al. Epidermal growth factor receptor mutations in lung cancer. Nat Rev Cancer 2007, 7(3):169-181

3. Hirsch FR, Varella-Garcia M, Dziadziuszko R, et al. Fluorescence in situ hybridization subgroup analysis of TRIBUTE, a phase III trial of erlotinib plus carboplatin and paclitaxel in non-small cell lung cancer. Clin Cancer Res 2008; 14(19):6317-23

4. Lynch TJ, Patel T, Dreisbach L, et al. A randomized multicenter phase III study of cetuximab $(\operatorname{Erbitux}(\mathrm{R}))$ in combination with Taxane/Carboplatin versus Taxane/Carboplatin alone as first-line treatment for patients with advanced/metastatic Non-small cell lung cancer (NSCLC): B3-03. J Thorac Oncol 2007; 2(8):S340-S341

5. Pirker R, Szczesna A, von Pawel J, et al. FLEX: A randomized, multicenter, phase III study of cetuximab in combination with cisplatin/vinorelbine $(\mathrm{CV})$ versus $\mathrm{CV}$ alone in the first-line treatment of patients with advanced non-small cell lung cancer (NSCLC) [abstract 3]. Proc Am Soc Clin Oncol 2008

6. Linardou H, Dahabreh IJ, Kanaloupiti D, et al. Assessment of somatic k-RAS mutations as a mechanism associated with resistance to EGFR-targeted agents: a systematic review and meta-analysis of studies in advanced non-small-cell lung cancer and metastatic colorectal cancer. Lancet Oncol 2008, 9(10):962-72

7. Therasse P, Arbuck SG, Eisenhauer EA, et al. New guidelines to evaluate the response to treatment in solid tumors. J Natl Cancer Inst 2000; 92:205-216.

8. Pao W, Chmielecki J. Rational, biologically based treatment of EGFR-mutant non-small-cell lung cancer . Nat Rev Cancer 2010; 10: $760-774$

9. Tang $X$, Shigematsu H, Bekele BN, et al. EGFR Tyrosine Kinase Domain Mutations Are Detected in Histologically Normal Respiratory Epithelium in Lung Cancer Patients. Cancer Res 2005, 65: 7568-7572

10. Eichler AF, Kahle KT, Wang DL, et al. EGFR mutation status and survival after diagnosis of brain metastasis in nonsmall cell lung cancer. Neuro Oncol. 2010 Nov;12(11):1193-9

11. Kalikaki A, Koutsopoulos A, Trypaki M, et al. Comparison of EGFR and K-RAS gene status between primary tumours and corresponding metastases in NSCLC. $\mathrm{Br} \mathrm{J}$ Cancer. 2008;99(6):923-9

12. Schmidt K, Oehl N, Wrba F et al. EGFR/KRAS/BRAF mutations in primary lung adenocarcinomas and corresponding locoregional lymph node metastases. Clin Cancer Res. 2009;15(14):4554-60.

13. Koo JS, Kim SH. EGFR and HER-2 status of non-small cell lung cancer brain metastasis and corresponding primary tumor. Neoplasma.2011;58(1):27-34 
14. Daniele L, Cassoni P, Bacillo E et al. Epidermal growth factor receptor gene in primary tumor and metastatic sites from non-small cell lung cancer. J Thorac Oncol. 2009 Jun;4(6):684-8.

15. Rami-Porta R, Crowley J, Goldstraw P. The Revised TNM Staging System for Lung Cancer. Ann Thorac Cardiovasc Surg 2009; 15: 4 - 9

16. Schmidt K, Oehl N, Wrba F et al. EGFR/KRAS/BRAF mutations in primary lung adenocarcinomas and corresponding locoregional lymph node metastases. Clin Cancer Res. 2009;15(14):4554-60.

17. Garcia-Olivé I, Monsó E, Andreo F et al. Endobronchial ultrasound-guided transbronchial needle aspiration for identifying EGFR mutations. Eur Respir J. 2010;35(2):391-5 